\title{
LAS "NUEVAS GUERRAS": UNA PROPUESTA METODOLÓGICA PARA SU ANÁLISIS
}

\author{
Víctor-Mario Bados Nieto ${ }^{1}$ y Marién Duran Cenit ${ }^{2}$ \\ Universidad de Granada
}

\begin{abstract}
Resumen:
Hoy día existen numerosos conflictos con características diferentes y singulares difícilmente encuadrables en los arquetipos de los conflictos armados tradicionales o guerras convencionales. De los muchos y variados apelativos surgidos principalmente desde el Fin de las Guerra Fría y como contraposición a las guerras clásicas, fue el concepto de "nuevas guerras" el que tuvo una mayor acogida. Sin embargo, como muchas otras denominaciones, consideramos que no podemos apreciarlo como una herramienta enteramente práctica para el estudio de toda la tipología de conflictos que existen. Por ello, el presente trabajo, mediante la revisión de la literatura más importante sobre estos temas, tiene como objeto proporcionar una herramienta teórico-metodológica, para que mediante la operacionalización de las variables identificativas de las "nuevas guerras", se puedan estudiar de forma más pormenorizada los conflictos contemporáneos para poder establecer comparaciones que arrojen luz sobre las similitudes y las diferencias.
\end{abstract}

Palabras clave: guerras, "nuevas guerras", conflicto étnico, monopolio de la violencia, estado fallido.

Title in English: "New Wars: A Methodological Proposal for their Analysis"

\begin{abstract}
:
There are many conflicts today with different and unique characteristics hardly categorized as traditional conventional wars or armed conflicts. Considering the varied denominations that can be found, mainly after the end of the Cold War, as opposed to conventional wars, it was "new wars" the name which had a better reception. However, like many other denominations, we believe that we cannot fully accept it as a practical tool for the study of all types of conflicts in the world. Therefore, this article, by reviewing the most important books and articles on this question, aims to provide a theoretical and methodological tool, making operational the identifying variables of the "new wars". Thus contemporary conflicts can be studied in detail in order to make comparisons that shed light on the similarities and differences.
\end{abstract}

Keywords: War, "new wars", ethnic conflict, monopoly of violence, failed state.

Copyright $\odot$ UNISCI, 2015.

Las opiniones expresadas en estos artículos son propias de sus autores, y no reflejan necesariamente la opinión de UNISCI. The views expressed in these articles are those of the authors, and do not necessarily reflect the views of UNISCI.

\footnotetext{
${ }^{1}$ Víctor Mario Bados Nieto es Teniente Coronel de Infantería Diplomado de Estado Mayor con diversos cursos militares, tanto nacionales como OTAN. Ha participado en diversas operaciones en el exterior y ha tenido destinos al mando de unidades de la Legión y en diversos Estados Mayores, tanto nacionales como internacionales.

E-mail: vbadnie@et.mde.es.

${ }^{2}$ Marién Duran Cenit es doctora europea en Ciencia Política y Profesora del Departamento de Ciencia Política y de la Administración de la Universidad de Granada.

Email: marienmduranc@ugr.es.
} 


\section{Introduction}

Podemos afirmar que hoy día existen numerosos conflictos que difieren por sus características y singularidad de aquellos que se han sucedido a lo largo de la historia. Fue con el fin de la Guerra Fría y durante los últimos años cunado han surgido múltiples apelativos a los cambios de matiz producidos en la naturaleza de la guerra: términos como "nuevas guerras" ${ }^{3}$, guerras de cuarta generación $(4 \mathrm{GW})^{4}$, guerras del tercer tipo ${ }^{5}$, guerras asimétricas ${ }^{6}$, guerra de espectador deportivo ${ }^{7}$, guerras híbridas ${ }^{8}$, guerra degenerada o guerra entre la gente ${ }^{9}$, se pusieron de moda y siguen siendo objeto de análisis en las principales investigaciones sobre estas materias. Todas estas etiquetas muestran que, además de la guerra en sentido clásico o convencional, surgieron más opciones y rostros diferentes de los conflictos en las últimas décadas ${ }^{10}$. Numerosos estudiosos e investigadores de diferentes disciplinas como la Seguridad (Kaldor $^{11}$, Duffield ${ }^{12}$ ), la Economía (Collier y Hoeffler ${ }^{13}$, Jung ${ }^{14}$ ), la Ciencia Política (Münkler ${ }^{15}$ ), la Sociología (Shaw ${ }^{16}$, Bauman ${ }^{17}$ ) o las Relaciones Internacionales (Holsti ${ }^{18}$, Bellamy ${ }^{19}$ ) coinciden en la existencia de un cambio en el fenómeno de la guerra y los conflictos armados tras la caída del Muro de Berlín. Todos ellos apuntan hacia unas nuevas señas de identidad que hacen que los nuevos conflictos surgidos a finales de siglo XX y que siguen hoy vigentes muchos de ellos, difieran como un todo de los hasta entonces acontecidos.

En este sentido, se hace necesario acudir a métodos comparados apropiados para poder afirmar que realmente existen diferencias entre los conflictos del pasado y los conflictos contemporáneos, y con ello elaborar diferentes categorías. La comparación es una de las

\footnotetext{
${ }^{3}$ Kaldor, Mary (2001): New and Old Wars: Organized Violence in a Global Era, Stanford, Stanford University Press.

${ }^{4}$ Lind, William S.; Nightengale, Keith; Schmitt, John F.; Sutton, Joseph W.; Wilson, Gary I.: "The Changing Face of War: Into the Fourth Generation", Military Review, vol. 69, nº 10 (1989), pp. 2-11.

${ }^{5}$ Holsti, Kalevi J. (1996): The State, War and the State of War, Cambridge, Cambridge University Press.

${ }^{6}$ Este término cobró importancia tras los atentados del 11S. En el año 1995 aparece la primera mención moderna al concepto de guerra asimétrica en un documento del Pentágono: la Joint Doctrine de 1995. En el año 1996, el general Ronald R. Fogelman habló sobre "una nueva forma americana de hacer la guerra". En el año 1999, el general norteamericano Wesley Clark durante la intervención de la OTAN en Kosovo lo puso de moda tras un artículo publicado en la revista Time.

${ }^{7}$ McInnes, C. (2006): Spectator-Sport War: The West and Contemporary Conflict, Colorado, Boulder Lynne Rienner.

${ }^{8}$ Hoffman Frank. (2007): Conflict in the 21st Century. The Rise of the Hybrid Wars, Arlington, Potomac Institute for Policy Studies.

${ }^{9}$ Shaw, Martin: "War and Globality: the role and character of war in the global transition", en Ho-Won Jeong (ed.), (1999): Peace and Conflict. A New Agenda, Hampshire, Ashgate Publishing.

${ }^{10}$ Münkler, Herfried (2005): Viejas y "nuevas guerras". Asimetría y privatización de la violencia. Madrid, Siglo XXI.

${ }^{11}$ Kaldor (2001), op. cit.

12 Duffield, M. (2004): Las "nuevas guerras" en el mundo global, Madrid, Catarata, Serie Relaciones Internacionales.

${ }^{13}$ Collier y Hoeffler: "Greed and grievance in civil war", Oxford Economics Papers, vol. 56, nº 4 (2004), pp. 563-595.

${ }^{14}$ Jung D. (2003): Shadow Globalization, ethnic conflicts and new wars. A political economy of intra-estate war, Nueva York, Routledge.

${ }^{15}$ Münkler, op. cit.

16 Shaw M. (1999): War and globality: the role and character of war in the global transition, Hampshire, Ashgate Publishing.

${ }^{17}$ Bauman, Zygmunt: "Wars of the Globalisation Era", European Journal of Social Theory, vol. 4, $\mathrm{n}^{\mathrm{o}} 1$ (2001), pp. 11-28.

${ }^{18}$ Holsti, op. cit.

${ }^{19}$ Bellamy, A.: "The Great Beyond: Rethinking Military Responses to New Wars and Complex Emergencies", Defence Studies, vol. 2, nº 1 (2002), pp. 25-50.
} 
herramienta más útiles para poder avanzar y explicar las diferencias y similitudes entre cada unidad de estudio. Por ello, los académicos de las ciencias sociales siempre han intentado establecer referencias de comparación para incrementar el conocimiento de cada caso, organizar los resultados por categorías, y obtener explicaciones y generalizaciones. ${ }^{20}$ Así lo hicieron por ejemplo Kaldor y Münkler cuando iniciaron sus investigaciones.

De esta manera, autores como Kaldor, establecen su punto de partida y de comparación durante la Guerra Fría. Ella expone que los conflictos de este período, -que se denominaron conflictos de baja intensidad para referirse a la guerrilla o al terrorismo-, constituyen el origen de lo que ella califica como las "nuevas guerras" de la posguerra Fría, aunque con características distintivas. Otros autores como Münkler se sitúan sin embargo en la Edad Moderna, pasando por la guerra de los Treinta Años e incluso por conflictos interestatales de nuestro siglo (China y Vietnam, Irak e Irán o la de Etiopía y Eritrea) para captar las diferencias y las peculiaridades de las "nuevas guerras".

Así, el término de "nueva guerra" se convirtió en una de las acepciones más utilizadas desde que apareció hace quince años. Pero quizá, en esta categoría (como también en otras) aunque se haya utilizado la comparación como referencia, no se ha operacionalizado lo suficiente para disponer de una herramienta más práctica, concisa y granular. Los trabajos realizados desde entonces se han centrado en analizar las características de estos conflictos de forma cualitativa, o en verter críticas hacia el concepto de nueva guerra, pero es interesante también poseer un instrumento para el análisis de estos conflictos.

Este trabajo propone por ello, partiendo del análisis de los trabajos sobre nuevas guerras, una herramienta teórica y metodológica para abordar los estudios de caso, que susceptibles de análisis u observación, puedan enmarcarse en el concepto o en la categoría de "nuevas guerras". Esta herramienta adopta una estrategia bipolar, esto es, "nuevas guerras" o lo contrario a ellas, esto es, guerra convencional y mediante un estudio empírico consigue captar los cambios cualitativos que han tenido lugar en la naturaleza del conflicto armado, sirviendo como guía para investigar la granularidad de los datos. Así, tras la identificación de las variables explicativas de las "nuevas guerras", se proponen una serie de indicadores y fuentes que pueden facilitar el trabajo al investigador que se acerque al estudio de caso de algún conflicto con el objeto de su categorización como "nueva guerra".

Para alcanzar este objetivo, el trabajo se organiza de la siguiente forma: en primer lugar se explican algunas de las contribuciones teóricas más importantes sobre las "nuevas guerras" y su origen, así como sus detractores; en segundo lugar, se analizan las variables identificativas de las nuevas guerras expuestas por diversos autores y gracias a la aportación de la revisión teórica; seguidamente, se operacionalizan las variables explicativas seleccionadas trasladándolas a una herramienta de análisis y disección de conflictos y por último se ofrecen unas conclusiones en las que se sintetiza el concepto de nuevas guerras y la herramienta propuesta para el análisis de los conflictos contemporáneos.

\section{Contribuciones teóricas sobre las "nuevas guerras"}

La finalidad de este apartado es efectuar un análisis sobre las contribuciones teóricas de las nuevas guerras. Tratamos en primer lugar las aportaciones de los autores que defienden la existencia de diferencias entre los conflictos contemporáneos y las guerras tradicionales. Principalmente tenemos las aportaciones de Lind, Van Creveld, Kaldor, Münkler, Duffield y Bellamy. En segundo lugar tratamos a los autores que rebaten a los "nuevos guerristas" como

\footnotetext{
${ }^{20}$ Landman, Tod (2011): Política comparada. Una introducción a su objeto y métodos de investigación, Madrid, Alianza Editorial, p. 28.
} 
Newman, Kalyvas, Henderson, Singer, Harbom, Wallensteen, Melander, Oberg, Hall y Gleditsch; y por último, consideramos la reciente respuesta de Kaldor a estos trabajos, defendiendo su hipótesis de las nuevas guerras. El objetivo de este análisis es extraer todas aquellas señas de identidad que a efectos metodológicos serán consideradas como variables independientes e identificativas claves sobre estos conflictos, ya trabajadas por los diferentes estudiosos.

El término "nuevas guerras" fue acuñado por Mary Kaldor ${ }^{21}$ tras analizar ampliamente las señas de identidad de los conflictos armados surgidos tras el final de la Guerra Fría si bien, académicos anteriores a ella, como Van Creveld y Lind ya apuntaron unas nuevas características que los nuevos conflictos estaban mostrando.

Así, Van Creveld aporta la tesis de que la guerra ha sufrido una transformación en el sentido de que pasa a ser una actividad que deja de perseguir unos objetivos racionales para convertirse en un fenómeno irracional como consecuencia del menoscabo de la legitimidad de los Estados. La guerra de esta manera, pierde su propósito político en el sentido clauwitziano y pasa a estar impulsada por otros de orden religioso, cultural, étnico o tecnológico.

Será posteriormente Lind quien acuñe el término de guerras de cuarta generación (4GW), como aquellas que usarán todas las redes y recursos disponibles en los ámbitos político, económico, social y militar para convencer a los que han de tomar las decisiones políticos del bando enemigo que, bien sus objetivos son inalcanzables, bien son demasiado costosos en cuanto a los beneficios a conseguir. Por ello las $4 \mathrm{GW}$ serán largas en cuanto a su duración.

En cuanto a las propuestas de Mary Kaldor, ésta basó sus estudios de caso en Bosnia y en Nagorno-Karabaj, considerando el primero como un caso paradigmático de "nueva guerra" El argumento fundamental que defendía es que "durante los años ochenta y noventa se desarrolló un nuevo tipo de violencia organizada -especialmente en África y Europa del Estepropio de la actual era de la globalización". Este tipo de violencia lo califica de "nueva guerra", utilizando el término "guerra" para subrayar "el carácter político de este nuevo tipo de violencia", si bien también reconoce que en estas "nuevas guerras" no están tan claros los motivos políticos: hay un desdibujamiento de límites en la guerra, adquiriendo importancia la presencia del crimen organizado ejercido por grupos privados y las violaciones a gran escala de los Derechos Humanos, no solo por los estados, sino también por grupos organizados políticamente $^{22}$.

Este tipo de violencia, muestra además notables diferencias con las guerras hasta entonces acaecidas. Estas diferencias se concretan en el origen de su aparición (la importancia de la globalización y de la internacionalización del conflicto); el marco geográfico en el que se desarrollan (ahora predominan más los conflictos intraestatales); los motivos que las animan (carácter identitario étnico o religioso); los métodos y estrategias de combate (violencia en algunos casos extrema contra civiles) formas de financiación (o lo que se conoce como nueva economía de guerra) y los actores que intervienen (fragmentación y multiplicidad de actores, privatización de la violencia).

En primer lugar, Kaldor defiende que son guerras propias de la era de la globalización. Son guerras locales y civiles en su mayoría, con múltiples repercusiones transnacionales. Esto es, son conflictos intraestatales en los que se produce una internacionalización del conflicto. Estas repercusiones implican una presencia internacional que incluye a todo un "ejército" internacional que va desde periodistas extranjeros, soldados mercenarios y asesores militares,

\footnotetext{
${ }^{21}$ Véase Kaldor (2001), op. cit., p. 15.

${ }^{22}$ Ibid., p. 15-16.
} 
expatriados voluntarios, ONG, instituciones internacionales, como la ACNUR, la UE, UNICEF, la OSCE y la propia ONU, mostrando, en definitiva, un tipo de respuesta a la guerra que se caracteriza por el cosmopolitismo.

Otra de las características que les atribuye, opuesta a las antiguas guerras civiles es la ideología versus identidad, al caracterizarse principalmente por movilizaciones que tienen su base en las cuestiones de identidad en contraste con las ideologías o geopolíticas de las antiguas guerras. Ella misma resalta que con política de identidades se refiere "a la reivindicación del poder basada en una identidad concreta: nacional, de clan, religiosa o lingüística. El peligro de este tipo de política reside en que fragmenta y excluye. Generan minorías diferenciadas, provocando discriminación psicológica y en casos extremos expulsión de poblaciones y genocidio.

En cuanto a los métodos de combate son guerras con y para la población versus violencia contra la población. Se caracterizan por una violencia extrema contra los civiles, con técnicas de guerrilla y contraguerrilla y desplazamientos masivos de población civil.

Y por último tiene en cuenta el factor de la economía de guerra. Las antiguas guerras eran más autárquicas y centralizadas, mientras ahora se han caracterizado por una nueva economía de guerra globalizada. En este sentido, una característica importante ha sido la privatización de la violencia. Sin embargo, en la práctica, como apunta Kaldor, la distinción entre lo privado y lo público, lo estatal y lo no estatal, lo informal y lo formal, lo que se hace por motivos económicos o políticos no es fácil de establecer. ${ }^{23}$

A la tesis sostenida por Kaldor se le sumarán numerosos estudiosos como Münkler, Bellamy, Beck, o Duffield, que aunque con algunas discrepancias, coinciden con la autora en la aparición de señas de identidad propias o claves de estos conflictos.

Es decir, unos rasgos diferenciadores que se contraponen a las características de las guerras clásicas y convencionales entre estados, las denominadas "viejas guerras". Estos rasgos diferenciadores hay que buscarlos sobre todo en el origen de estos conflictos. Conocer la génesis de los mismos y su contextualización es fundamental para saber interpretarlos y diferenciarlos de otros tipos de conflictos.

En general, los principales motivos aportados por diversos autores como desencadenantes de las "nuevas guerras", los podemos cifrar en los siguientes: la globalización, la finalización de la Guerra Fría y la crisis del estado westfaliano.

En primer lugar y siguiendo fundamentalmente a Kaldor, considera a la globalización como causa primaria en la aparición de las "nuevas guerras". De hecho, introduce su obra afirmando que "las "nuevas guerras" deben interpretarse en el contexto del proceso conocido como globalización", entendiendo ésta como una "intensificación de las redes globales, militares y culturales a escala mundial... como una consecuencia de la revolución en las tecnologías de la información y también de las drásticas mejoras en la comunicación y el tratamiento de datos" 24 . ¿Cómo se aprecia el impacto de la globalización en las "nuevas guerras"? Este impacto se visibiliza en la presencia de una diversidad de nuevos actores civiles y militares cuanto menos no tan numeroso como en las guerras precedentes.

Otros autores como Duffield, Ishiyama, Bellamy, Hobsbawm y Bauman, también consideran a la globalización como origen de los nuevos conflictos. Duffield, aprecia con la globalización un nuevo patrón entre las políticas de los países del Norte que pone un nuevo énfasis en la falta de desarrollo en el Sur al "fomentar la inestabilidad internacional a través

\footnotetext{
${ }^{23}$ Ibid., pp. 15-28.

${ }^{24}$ Ibid., pp. 17-18.
} 
del conflicto, las actividades delictivas y el terrorismo" ${ }^{25}$. Ishiyama contempla a la violencia entre iguales étnicos y sociales como una forma de tratar de arreglar o estabilizar la identidad étnica en medio de las incertidumbres de la globalización. ${ }^{26}$ Bellamy, por el contrario, reconoce a la globalización como una creciente interconexión de las sociedades que ha tenido efectos innegables sobre la capacidad de los grupos rebeldes para difundir su mensaje y buscar adeptos y financiación ${ }^{27}$. Hobsbawm afirma que la globalización es la forma actualmente dominante del capitalismo de libre mercado que ha traído un aumento considerable de las desigualdades sociales y económicas dentro de cada país e a nivel internacional. ${ }^{28}$ En términos similares se expresa Bauman al sostener que hay una serie de consecuencias negativas de la globalización, tales como la generación de excedentes que no tienen a dónde ir en un mundo repleto, con desigualdades cada vez más visibles en un mundo en el que cada vez es más difícil garantizar la seguridad colectiva para las comunidades y las naciones. $^{29}$

La segunda causa que se apunta como facilitadora de las "nuevas guerras" la encontramos en la finalización de la Guerra Fría. Sin embargo esta causa para Kaldor es a su vez consecuencia de que el Bloque del Este sucumbiera a la globalización. Por tanto, no la podemos contemplar enteramente como una causa originaria. Lo que sí se puede afirmar, es que la caída del Muro de Berlín produjo una distribución de poder menos estable que la resultante de tener un orden mundial bipolar. También Kaldor argumentará que las "nuevas guerras" son consecuencia indudable de la reconfiguración del poder resultado del final de la Guerra Fría, lo que permitió la disponibilidad de excedentes de armas sin control, el descrédito de las ideologías socialistas, la desintegración de los imperios totalitarios y la retirada de apoyo de las superpotencias a regímenes clientelares. ${ }^{30}$ Para Snow, el sistema internacional altamente estructurado y predecible en sus relaciones colapsó y evolucionó hacia un mundo más desregulado. Las grandes potencias no sentían ni apetito, ni tenían capacidad para controlar la violencia interna en los países del Tercer Mundo. ${ }^{31}$

Siguiendo de nuevo a Kaldor, igualmente otro de los motivos principales que ha desencadenado su aparición, lo podemos encontrar en lo que se puede enmarcar como la crisis de los estados westfalianos surgidos tras $1648 .{ }^{32}$ Según la autora, los estados se sintieron impelidos a monopolizar el uso de la violencia y eliminar con ello la violencia organizada, así como a los demás actores privados surgidos en los conflictos. Si se falla en esta labor, el estado pierde su legitimidad, como diría Carl Schmitt "Protect ergo obligo, es decir, mientras proteja tendré la obediencia de los súbditos. De este modo, las "viejas guerras" quedan asociadas a los estados nación. Eran conflictos entre estados con clara diferenciación de lo público y lo privado, los asuntos internos de los externos y la política de la economía. ${ }^{33} \mathrm{~A}$ este argumento se sumarán también otros autores quienes apelarán al surgimiento de los estados fallidos en los que aparecerá el fallo de la modernidad. ${ }^{34}$ Consideran que la exclusión del Sur del capitalismo global ha acrecentado las diferencias con los países pobres, y permitido el

\footnotetext{
${ }^{25}$ Duffield (2004), op. cit.

${ }^{26}$ Ishiyama, John.: "Does Globalization Breed Ethnic Conflict?", Nationalism and Ethnic Politics, vol. 9 , n 4 (2004), pp. 1-23.

${ }^{27}$ Bellamy, op. cit., pp. 25-50.

${ }^{28}$ Hobsbawn, Eric (2006): Guerra y Paz en el siglo XXI, Barcelona, Crítica.

${ }^{29}$ Bauman, op. cit., pp. 11-28.

${ }^{30}$ Véase Kaldor (2001), op. cit., p. 18.

${ }^{31}$ Snow, Donald (1996): Uncivil wars: international security and the new internal conflicts, Boulder, Lynne Rienner publishers.

${ }^{32}$ Véase Kaldor (2001), op. cit.

${ }^{33}$ Kaldor, Mary: "A decade of Humanitarian Intervention: the role of Global Civil Society", en Glasius, M.; Kaldor, M. \& Anheier, H. (ed.) (2002): Global Civil Society, Oxford, Oxford University Press, pp. 109-148.

${ }^{34}$ Véase Bauman, op. cit., pp. 11-28.
} 
colapso de estructuras estatales (estados fallidos) y la aparición de gobiernos débiles y corruptos. Münkler también alude a la pérdida del monopolio de la violencia por parte del estado, quien ya no garantiza la seguridad de sus súbditos. ${ }^{35}$ Igualmente Ulrich Beck se une a estas tesis, afirmando que se ha producido una desfronterización de la guerra, producto en primer lugar de la pérdida del monopolio legítimo de la violencia del estado (aparición de señores de la guerra, compañías privadas de seguridad, ejércitos privados, etc.) y por otro lado de la adquisición de conciencia de la globalidad de los peligros, lo cual también puede tener su vertiente positiva puesto que aumenta la conciencia de los Derechos Humanos, y los estados en algunas ocasiones se unen para ponerle fin ${ }^{36}$.

Sin embargo, como contestación a estas tesis, aparecen otros estudiosos que se oponen a la existencia de esta nueva realidad. Entre ellos destacan Newman, Kalyvas, Henderson, Singer, Harbom, Wallensteen, Melander, Oberg, Hall y Gleditsch como más significativos, aunque nos centraremos en las tesis de los dos primeros. Consideran esencialmente que no se debe hablar de "nuevas guerras" como fenómeno que presente rasgos identificativos diferentes de los conflictos conocidos hasta el momento. Según estos autores la distinción es exagerada, ya que no sólo la realidad social de los conflictos armados ha cambiado, sino también nuestro enfoque y análisis. Para ello recurren fundamentalmente a técnicas cuantitativas para demostrar que las conclusiones, principalmente cualitativas de los "nuevos guerristas" son, bien erróneas, bien distorsionadas.

Newman rebate desde los datos que las "nuevas guerras" no presentan elementos cualitativos muy distintos de las hasta ahora existentes (intraestatales, conflictos étnicos y religiosos, mayor cantidad de bajas civiles, privatización de la violencia, presencia de actores estatales y no estatales, grupos terroristas, colapso de la autoridad pública etc.). Es más, después de analizar los argumentos de las "nuevas guerras", defiende que las diferencias entre estos conflictos y las guerras tradicionales se han exagerado y que esta tendencia ha olvidado incluso que en los conflictos contemporáneos también existen diferencias entre ellos. Apunta que lo que ha variado es nuestra aproximación y análisis en el estudio de los conflictos. También defiende que la realidad ha cambiado, pero no tanto como la presenten los defensores de las tesis de las "nuevas guerras". Para él, muchas de las características de estos conflictos no son nuevas, sino que han estado presentes con una intensidad diferente en los últimos cien años: actores, objetivos, contexto espacial, impacto humano, economía política y estructura social del conflicto, son variables que no han cambiado tanto. La diferencia hoy día subyace en los factores que los que se mantiene la focalización y en el papel de los medios de comunicación ${ }^{37}$

Kalyvas considera que la distinción actual entre "nuevas" y "viejas" guerras civiles es errónea. La base del argumento, al igual que Newman, es que las características de las "nuevas guerras" han sido exageradas y no estudiadas con exhaustividad y que las "viejas guerras" han estado malinterpretadas y desconsideradas. Propone una visión micro, es decir, buscar la granularidad del problema en vez de atender solo a los aspectos macro que debilitan el argumento. Defiende en este sentido que una buena teoría sobre las guerras civiles requiere de categorías conceptuales y de indicadores empíricos fiables. Para ello, utilizando y haciendo acopio de investigación etnográfica pretende demostrar que la información sobre las guerras civiles contemporáneas es incompleta y que por otro lado, se le ha prestado poca atención a las investigaciones que afectan a las guerras civiles del pasado. Para hacer la comparación utiliza tres dimensiones: (i) causas y motivaciones; (ii) apoyos; (iii) y violencia. Mediante su

\footnotetext{
${ }^{35}$ Véase Münkler, op. cit.

${ }^{36}$ Beck, Ulrich (2005): La Mirada Cosmopolita o la guerra es la paz, Barcelona, Paidós Estado y Sociedad.

37 Newman, Edward: "The New wars debate: A historical perspective is needed", Security Dialogue, vol. 35 (2004), pp. 137-189.
} 
análisis rompe con los esquemas de las diferencias que se han argumentado en estas dimensiones. Así por ejemplo sobre si los nuevos conflictos están motivadas por odios tribales y étnicos o botines privados frente a las viejas guerras civiles en las que se luchaba por causas nobles como el cambio social y en las que los motivos de reclamación eran colectivos, Kalyvas afirma que en muchos casos la ideología en otros conflictos es menos visible a los ojos occidentales al estar basada en perspectivas culturales locales. En sentido parecido aborda los apoyos (apoyo popular o falta de apoyo), la violencia (gratuita o controlada), la avaricia o el saqueo como causas de los mismos. ${ }^{38}$

Otros autores como Lacina ${ }^{39}$, Harbom y Wallensteen ${ }^{40}$, Lacina y Gedistch ${ }^{41}$ se centran $^{2}$ en los datos sobre la violencia de los conflictos, tanto en los niveles de atrocidad, como en el número y naturaleza de las pérdidas humanas. Así, demuestran que no ha habido un aumento significativo en el número de muertes y que la relación de muertes de militares y civiles no ha cambiado de manera importante. Por ejemplo, Lacina argumenta que la democracia, más que el desarrollo económico o la fortaleza militar del estado, está más fuertemente correlacionada con menos muertes. En este sentido, al expandirse la democracia, las guerras han sido menos mortíferas desde el final de la Guerra Fría.

A estos estudiosos detractores de los "nuevos guerristas", saldrá nuevamente Kaldor en el año 2013 a rebatir sus propuestas.

Comienza su análisis haciendo una revisión de los términos propuestos por otros autores que confieren adjetivos al concepto de guerra para reafirmarse en el hecho de que los conflictos contemporáneos son diferentes de los conflictos tradicionales. Para ello también efectúa en su ensayo un análisis de los cuatro principales bloques en los que se centran las críticas o los elementos del debate: la lógica de las nuevas guerras; si las nuevas guerras son nuevas; si las nuevas guerras son guerras; y el debate sobre los datos y el debate sobre Clausewitz. En el primer apartado insiste en la diferencia entre viejas y nuevas guerras en cuestiones como los actores, los objetivos, los métodos y las formas de financiación ${ }^{42}$. En el segundo y siguientes apartados se centra más en la parte conceptual. En esta parte conceptual, la autora defiende el adjetivo "nuevo" en el sentido de que debe entenderse como una forma de esclarecer la lógica de los conflictos contemporáneos que puede ofrecer, tanto una estrategia de investigación, como una guía para la política.

Como estrategia de investigación es indudable que el debate sobre las nuevas guerras, abrió en su momento nuevas perspectivas de análisis, contribuyendo a enriquecer el campo de los estudios sobre los conflictos. La evidencia está en los múltiples trabajos que se han desarrollado en estas décadas, tanto de carácter más cualitativo como incluso cuantitativo teniendo como referencia en estos últimos las fuentes de datos que cita Kaldor como el Uppsala Conflict Data Programma (UCDP) de la Universidad de Uppsale; "Correlates of War Project de la Universidad de Michigan y "The biennial Peace and Conflict Survey, elaborado por el Centro para el Desarrollo y la Gestión del Conflicto de la Universidad de Maryland

Como guía para la política es también importante señalar los debates y trabajos que se han desarrollado dentro de las organizaciones militares, las organizaciones internacionales

\footnotetext{
${ }^{38}$ Kalyvas, Stathis N.: "New and Old Civil Wars. A valid distinction?", World Politics, vol. 54 (2001), pp. 99118.

${ }^{39}$ Lacina, Bethany: "Explaining the severity of civil wars", Journal of Conflict Resolution, vol. 50, n 2 (2006), pp. 276-289,

${ }^{40}$ Harbom, Lotta \& Wallensteen, Peter: "Armed conflict and its international dimensions, 1946-2004", Journal of Peace Research, vol. 42, no 5 (2005), pp. 623-635.

${ }^{41}$ Bethany Lacina Nils Petter Gledistch: "Monitoring Trends in Global Combat: A New Dataset of Battle Deaths", European Journal of Population, vol. 21, no 2-3 (2005), pp. 145-166.

${ }^{42}$ Véase Kaldor, Mary: "In Defence of New Wars", Stability, vol. 2, no 1 (2013), p. 2.
} 
como la OTAN, la Unión Europea o Naciones Unidas, los ministerios de defensa. Los conflictos contemporáneos han abierto múltiples discusiones en todos estos ámbitos, no solamente sobre su naturaleza, conceptualización o denominación, sino también sobre todo lo que llevan aparejado. Así ha sido común al hilo de estos conflictos escribir y hablar sobre la seguridad humana, la contrainsurgencia, la conciencia intercultural, la identidad, actores no estatales, emergencias complejas, las relaciones civiles-militares, etc. ${ }^{43}$

De esta manera y, desde nuestra perspectiva, en este trabajo se entiende que lo importante son las tendencias que conforman características comunes que definen tipologías, y en ese sentido se coincide con los "nuevos guerristas" en que en las últimas décadas se están produciendo conflictos con unas similitudes entre ellos muy pronunciadas y que a la vez se diferencias de los conflictos tradicionales y de los cuales podemos extraer una serie de variables identificativas. Como apunta Kaldor ${ }^{44}$, se debe subrayar la lógica de las nuevas guerras y aquí está muy claro que los actores de los conflictos contemporáneos son muy diferentes de los tradicionales; igualmente los objetivos son diferentes (geopolítica e ideología en los conflictos tradicionales, frente a identidad en los contemporáneos); los métodos difieren también de manera indiscutible sobre todo hacia quién se dirige la violencia; y por último las formas de financiación (financiación de los estados versus otras formas que incluyen múltiples facetas). También según Kaldor, aunque los datos deben tomarse con cautela, nos ofrecen un apoyo inigualable en el estudio de estos conflictos para defender la tesis de las nuevas guerras ${ }^{45}$. Lo importante en definitiva es considerar estas características en las "nuevas guerras" como una herramienta teórico-metodológica para entender mejor sus lógicas de gestación y desarrollo.

\section{Análisis de las variables identificativas de las "nuevas guerras"}

Una revisión profunda de la literatura existente sobre las nuevas guerras, nos ha proporcionado una serie de elementos identificativos claves sobre las mismas. Nuestro trabajo, ha consistido de esta forma en identificar las principales señas de identidad de los nuevos conflictos que aportan los distintos autores. Estas nuevas señas identificativas de las nuevas guerras serán consideradas como variables independientes que posteriormente estarán sujetas a una operacionalización para extraer los indicadoes cuantitativos que nos permitan unas conclusiones válidas. En primer lugar, Kaldor, como la autora que se ha dedicado más pormenorizadamente a esta cuestión, establece cuatro áreas que analiza de forma exhaustiva: actores, causas, métodos y estrategias empleadas y formas de financiación. ${ }^{46}$ A éstas cuatro se añadirán otras tres categorías que se denominarán emergencias complejas, marco geográfico y marco legítimo- legal de la violencia. Con estas siete categorías se pretende captar y encapsular todas las características de los nuevos conflictos como paso previo a la síntesis de las variables explicativas de los mismos.

\subsection{Actores}

Son muchos los estudiosos que coinciden en la gran multiplicidad y fraccionamiento de los actores en los nuevos conflictos. ${ }^{47}$ Kaldor pone de manifiesto la existencia de multitud de actores, públicos y privados, estatales y no estatales, muchos de ellos sin uniforme, hecho que

\footnotetext{
${ }^{43}$ Kaldor (2013), op. cit., pp.1-16.

${ }^{44}$ Ibid.

${ }^{45}$ Ibid., p. 2 y 3.

${ }^{46}$ Véase Kaldor (2001), op. cit.

${ }^{47}$ Veáse por ejemplo Bellamy, op. cit.; Snow, op. cit.; Kaldor (2001), op. cit.; Eppler, Erhard (2002) Vom Gewaltmonopol zum Gewaltmarkt? Die Privatisierung und Kommerzialisierung der Gewalt, Frankfurt/M.: Suhrkamp; Münkler, op. cit.
} 
en parte puede ser explicado por la mayor criminalización de las economías de guerra. Establece cinco principales tipos de actores: fuerzas armadas regulares o restos de las mismas; grupos paramilitares; unidades de auto-defensa; mercenarios extranjeros y finalmente fuerzas regulares extranjeras, generalmente bajo auspicios internacionales ${ }^{48}$. Considera que las fuerzas regulares están en declive, particularmente en las zonas de conflicto, las cuales sufren de recortes, con pérdida de prestigio, escasez de equipo y piezas de repuesto, entrenamiento inadecuado y pérdida de moral. Son las fuerzas paramilitares las más comunes en los conflictos y las que operan autónomamente alrededor de un líder, aunque también hace hincapié en la creciente importancia de las empresas de seguridad privada y los niños soldados. 49

Por otra parte Duffield entiende estas "nuevas guerras" como las "guerras en red", es decir guerras que se basan en redes cada vez mas privatizadas de actores estatales y no estatales que actúan mas allá de las competencias territoriales de gobiernos definidos territorialmente. Esto significa que en vez de ejércitos o estados, en estas guerras se alían redes políticas, sociales o económicas (Duffield 2001). Para Münkler, existen muchos nuevos actores protagonistas de las "nuevas guerras": actores paraestatales y en parte privados, señores de la guerra, grupos de guerrilleros locales, empresas de mercenarios. Monika Heupel y Barnhard Zangl confirman estas observaciones añadiendo que en la guerra de BosniaHerzegovina llegaron a verificarse hasta 83 diferentes unidades de combate, que iban desde desertores del ejército regular, paramilitares, mercenarios extranjeros, milicias locales o bandas criminales. ${ }^{50}$ Estos actores además adolecen de una sólida estructura y cadena de mando jerarquizada, con gran atomización de los grupos de combatientes que responden más bien a estructuras horizontales que operan autónomamente.

\subsection{Causas}

De las causas que los estudiosos apuntan como desencadenantes de las "nuevas guerras", un primer grupo gira en torno a las identidades políticas basadas en cuestiones religiosas, étnicas y tribales, mientras que en un segundo elenco se encuentran aquellas otras motivadas por la contienda por los recursos.

En cuanto a las relacionadas con las identidades, Kaldor apunta como principal característica de las "nuevas guerras" lo que denomina las identidades políticas. Asegura que, en las "viejas guerras" se dirimían los asuntos de orden geopolítico o se combatía por las ideologías o por los territorios. Sin embargo en los nuevos conflictos, los elementos que entran en liza serán aquellos relacionados con las identidades políticas, entendiendo éstas como aquellas doctrinas que tratan de fortalecer y hacer hincapié en la importancia de las estructuras de poder basadas en la tribu, la nación, el clan, de religión o la lengua. Y aun cuando algunos analistas lo consideran un hecho basado en raíces históricas, Kaldor aprecia claramente este nuevo nacionalismo como de reciente construcción al contener dos nuevas señas de identidad. Por un lado busca la desintegración del estado y no la construcción del mismo y por otro, es un nacionalismo carente de ideología modernizadora. ${ }^{51}$

A diferencia de la política de las ideas, que está abierta a todos y, por tanto, tiende a ser integradora, este tipo de política de identidades es intrínsecamente excluyente y, por tanto, tiende a la fragmentación. Pero la nueva ola de identidad es a la vez local y mundial, nacional

\footnotetext{
${ }^{48}$ Véase Kaldor (2001), op. cit.

${ }^{49}$ Kaldor (2002), op. cit., pp. 109-148.

${ }^{50}$ Heupel, Monika y Zangl, Barnhard (2010): "On the transformation of warfare: a plausibility probe of the new war thesis", Journal of International Relations and Development, vol. 13, nº 1 (March 2010), pp. 26-58.

${ }^{51}$ Véase Kaldor (2001), op. cit.
} 
y transnacional, utilizando las nuevas tecnologías para su movilización. ${ }^{52}$ En este contexto Creveld afirma también que la guerra no se libra como instrumento de la política, sino más bien como un instrumento de justicia, la religión o de supervivencia. ${ }^{53}$ Münkler se expresa en el mismo sentido cuando afirma que las guerras en Bosnia, el Cáucaso o Afganistán habrían transcurrido de modo distinto de no haber existido antagonismos de carácter étnico o religiosos. ${ }^{54}$ Bellamy coincide con Kaldor en el postulado de la identidad como causa desencadenadora de los nuevos conflictos, en los que la etnia y la religión juegan un papel fundamental. Tanta importancia ha adquirido esta variable que los estudios que se han desarrollado han sido sumamente detallados. Así las identidades como principal causa de los nuevos conflictos, queda refrendada por los datos proporcionados por el Stockholm International Peace Research Institute (SIPRI). En ellos se aprecia como el sesenta por ciento (60\%) de los conflictos no estatales que se libraron entre 2001 y 2010 fueron por cuestiones de carácter étnico o religioso 55 .

En el segundo grupo de principales causas que hemos de apuntar como constitutivas de las "nuevas guerras" se sitúan las de orden económico, más en concreto la contienda por la adquisición y tenencia de recursos. En ese sentido se expresa David Keen cuando llega a versionar el famoso aserto de Clausewitz definiendo a la guerra como la "continuación de la economía por otros medios". ${ }^{56}$ Cierto es que los estados están perdiendo relevancia a favor de los mercados. Münkler considera que en las "nuevas guerras", el uso de la fuerza se ha convertido en una fuente de ingresos, bien para procurarse subsistencias, bien para enriquecerse. De ahí la aparición de los señores de la guerra que controlan un territorio por las armas para explotar los recursos naturales o para proporcionar licencias de explotación. De este modo Münkler las califica como "largas guerras intrasociales y transnacionales", en las que la guerra llega en muchos casos a transformarse en una forma de vida para los actores implicados. $^{57}$

En esta interpretación de la búsqueda de recursos como causa de las "nuevas guerras", encontramos en los autores Collier y Hoeffler, el indicador de la codicia como impulso principal en los nuevos conflictos. En la lucha existente por los recursos, constituye la codicia el enfrentamiento por mero afán lucrativo de las partes. Los autores que se alinean con este argumento hacen hincapié en que el robo, el saqueo y toda forma de financiación criminal se convierten en el verdadero "leitmotiv" del conflicto, pasando a un segundo o tercer plano el papel que juegan las ideologías o la lucha política. Ambos sostienen que en realidad las condiciones de extrema pobreza no son suficientes para que estallen los conflictos, sino que además la oportunidad de beneficios económicos ha de ser muy alta, por lo tanto la razón no reside tanto en cuestiones de orden económico como en la codicia. ${ }^{58}$

\subsection{Métodos y estrategias}

Las características de los nuevos conflictos han originado que se cambie el modo de combatir, es decir, que se desarrollen nuevas aproximaciones en el ámbito de la táctica y la estrategia que difieren de los hasta ahora existentes. No quiere decir que no se hayan empleado en algún

\footnotetext{
${ }^{52}$ Ibid.

${ }^{53}$ Van Crevald, Martin (1991): The Transformation of War, New York, Free Press

${ }^{54}$ Véase Münkler, op. cit..

55 Lotta, Themnér y Wallensteen, Peter (2012): "Patterns of organized violence, 2001-10", en SIPRI Yearbook 2, Oxford, Oxford University Press.

${ }^{56}$ Keen, David: "The Economic Functions of Violence in Civil Wars", Adelphi Papers, $\mathrm{n}^{\circ} 320$ (1998), Routledge, p. 11.

${ }^{57}$ Véase Münkler, op. cit., pp. 19.

${ }^{58}$ Collier, Paul y Hoeffler, Anke: "Greed and grievance in civil war", Oxford Economics Papers, Vol. 56, no 4 (2004), pp. 563-595.
} 
momento en la historia, pero al menos no con la profusión y como finalidad como en las guerras del momento.

En ese sentido los diversos autores inciden en la aparición de una serie de características que nos conducen a pensar en la particularidad de los métodos y estrategias: una mayor violencia contra la población civil, el hibridismo de su naturaleza, la asimetría cada vez más profunda de los conflictos y la diferente tolerancia a las bajas.

La virulencia contra las poblaciones civiles en estos conflictos es especialmente problemática. De este modo, Kaldor atribuye como objetivo final de la táctica, las acciones que anteriormente eran resultantes de los daños colaterales. Así una de las características es el incremento de la violencia dirigida contra los civiles ${ }^{59}$ con genocidios, masacres, desplazamientos de poblaciones, violencia sexual contra las mujeres y violencia dirigida en muchos casos contra las minorías diferenciadas étnicamente.

El hibridismo de estos conflictos es otro indicador bastante significativo. Las nuevas estrategias bélicas aprovechan la experiencia tanto de la guerrilla como de la lucha contrarrevolucionaria, pero de modo peculiar. En las "viejas guerras" el objetivo era la captura del territorio por medios militares; las batallas eran los enfrentamientos decisivos. La guerra de guerrillas se desarrolló para sortear las grandes concentraciones de fuerza militar que caracterizaban a la guerra convencional. En las "nuevas guerras" sin embargo, el territorio se captura mediante el control político de la población. Se intentan evitar los combates todo lo posible. ${ }^{60}$ Las "nuevas guerras" toman prestadas de la contrarrevolución las técnicas de desestabilización dirigidas a sembrar «el miedo y el odio». El objetivo es controlar a la población deshaciéndose de cualquiera que tenga una identidad distinta, incluso una opinión distinta. Por eso, el objetivo estratégico de estas "nuevas guerras" es expulsar a la población mediante diversos métodos, como las matanzas masivas, los reasentamientos forzosos y una serie de técnicas políticas, psicológicas y económicas de intimidación. Ésa es la razón de que en todas estas guerras haya habido un aumento espectacular del número de refugiados y personas desplazadas, y de que la mayor parte de la violencia esté dirigida contra civiles. También esta es la razón por la que hay un mayor número de bajas civiles. En similar sentido se postula Smith cuando considera que la guerra como tal no existe y acuña el término de "Guerra entre la gente". Las guerras ya no se libran en el campo de batalla, con el fin de alcanzar un objetivo político a través de una batalla decisiva, sino que se cuenta con la presencia de actores no estatales que luchan en medio de la población. ${ }^{61}$ Es decir, en esta nueva forma de violencia resulta difícil distinguir lo político de lo económico, lo público de lo privado, lo militar de lo civil. ${ }^{62}$ Esta circunstancia de indefinición y desdibujamiento de límites entre las diferentes esferas de la guerra es lo que Hoffman denomina conflicto híbrido y que se manifiesta en la coexistencia de armamento, tácticas y combatientes muy heterogéneos ${ }^{63}$.

Otra de las características en los nuevos métodos y estrategias de combate, muy ligada a la anterior, es la existencia de una acusada asimetría que se ha producido en la aparición de estos nuevos conflictos. Este proceso se aceleró tras la "Revolución en los Asuntos Militares" que se inició hace más de veinticinco años y que ha incorporado la alta tecnología a los sistemas de armas. ${ }^{64}$ De esta manera, se categorizan de diferentes modos las asimetrías entre

\footnotetext{
${ }^{59}$ Véase Kaldor (2001), op. cit.

${ }^{60} \mathrm{Ibid}$.

${ }^{61}$ Smith, Rupert (2005): The Utility of Force - The Art of War in the Modern World, London, Allen Lane.

${ }^{62}$ Véase Kaldor (2001), op. cit.

${ }^{63}$ Hoffman, Frank (2007): Conflict in the 21st Century: The Rise of the Hybrid Wars, Arlington, Potomac Institute for Policy Studies.

${ }^{64}$ Véase Münkler, op. cit.
} 
los diversos actores, y que van desde actores estatales versus no estatales, la capacidad de los mismos (países OCDE versus resto del mundo), o la brecha existente entre los EEUU y sus aliados en la OTAN. ${ }^{65}$

Nos encontramos por tanto con conflictos en los que existe una clara disimilitud entre las partes enfrentadas en lo relativo al nivel tecnológico del armamento y material empleado, capacidad logística y de acceso a recursos, y nivel de instrucción y adiestramiento, lo que conduce a que se recurran a estrategias y tácticas de combate muy diferentes: guerrilla civil, pequeña guerrilla o "guerras del tercer tipo"66

De este modo y en cuanto a la desemejanza entre las partes aparecida en los nuevos con conflictos, Munkler argumentará que la aparición de las asimetrías surge como consecuencia de la incontestable superioridad económica, tecnológica y militar de los EEUU. Importante de resaltar es que esta asimetría ha ocasionado la aparición de una nueva estrategia políticomilitar para contestar tal superioridad, el terrorismo. Presenta Münkler tres tipos de guerras asimétricas: guerras por los recursos, guerras reguladoras y las guerras de desgaste. ${ }^{67}$

Como consecuencia se puede afirmar que esta asimetría, es decir, esta diferencia en recursos y tecnología entre los bandos oponentes ha sido la causa de la extensión del terrorismo como estrategia de combate para contrarrestar tal inferioridad. Kaldor afirma que el terrorismo debe ser entendido como una de las variantes de las "nuevas guerras" como resultado lógico de la evolución de las tácticas en los conflictos contemporáneos. ${ }^{68}$

Por último, otro de los aspectos que deben considerarse en la estrategia de combate es el relativo a la diferente tolerancia a las bajas. Münkler sostiene que las sociedades occidentales son "post-heroicas", pues tienden a aceptar una baja tolerancia a las bajas militares y las cargas económicas. Por lo tanto, plantean los conflictos cortos e intensos. Cuanto más tiempo dura una guerra, mayor será la probabilidad de que una sociedad "post-heroica" retire a sus tropas. ${ }^{69}$ En la misma argumentación se posiciona Luttwak, reforzando esta tesis al afirmar que, en aquellos conflictos en los que intervienen sociedades democráticas de países desarrollados occidentales, existe una muy baja de tolerancia a las bajas. A estas circunstancias se une el uso de la tecnología que les permite el llevar a cabo una guerra casi virtual. En el otro lado nos encontramos las sociedades menos desarrolladas, con una mayor tasa de natalidad, que tienen una disponibilidad superior a sacrificar. Por ello los ejércitos postmodernos deben, compensar su falta de disposición a sacrificarse mediante la tecnología, con el fin de poder derrotar a los contendientes a distancia ${ }^{70}$.

\subsection{Formas de financiación}

Las "nuevas guerras" también han traído consigo nuevas formas de financiación y con ella, una nueva economía de guerra. Kaldor y Münkler estudian en profundidad este aspecto. Las antiguas guerras eran más autárquicas y centralizadas, mientras ahora se han caracterizado por una nueva economía de guerra globalizada. Las formas tradicionales de financiación mediante la tasación y subida de impuestos de los estados y la creación de las economías de guerra han desaparecido. Las "nuevas guerras" tienen lugar en un contexto de extrema globalización, con

\footnotetext{
${ }^{65}$ Yost, DS: "The US-European capabilities gap and the prospects for ESDP", en Howorth J, Keeler JTS (eds.) (2003): Defending Europe. The EU, NATO and the Quest for European Autonomy, New York, Palgrave Macmillan, pp. 81-106.

${ }^{66}$ Holsti, op. cit.

${ }^{67}$ Véase Münkler, op. cit.

${ }^{68}$ Véase Kaldor (2001), op. cit.

${ }^{69}$ Véase Münkler, op. cit..

${ }^{70}$ Luttwak Edward N.: "A Post-Heroic Military Policy", Foreign Affairs, vol. 75, no 4 (julio-agosto 1996), pp. 33-44.
} 
un colapso del estado y la destrucción de sus estructuras. De este modo, las bases de tasación e impuestos colapsan, ya por las dificultades de su recaudación, ya por la caída de la producción. Asimismo los mercados se cierran a los estados colapsados mediante los bloqueos exteriores o por las fuerzas combatientes sobre el terreno. ${ }^{71}$ Las principales formas de financiación pasan a ser "la transferencia de los recursos" ${ }^{72}$ o mediante la ayuda exterior como consecuencia del colapso de la producción interna. Por una parte, la forma más simple de transferencia de recursos es el saqueo, el robo, la extorsión, el pillaje, rescates por rehenes, etc. Otra forma es la presión de los mercados, como el establecimiento de check-points para controlar los abastecimientos logísticos, los bloqueos y cercos, así como la división del territorio entre diferentes grupos paramilitares que permiten el control de los precios de mercado. Otro procedimiento más sofisticado de generar ingresos son los impuestos de guerra por la facciones o la exigencia de dinero para proporcionar protección tanto física como de producción de bienes. Por otra parte, la ayuda exterior se convertirá en crucial y tendrá lugar en forma de armamento, municiones y logística de todo tipo. Estas ayudas pueden llegar mediante transferencias de capital del extranjero en grupos, individuos de la diáspora, de gobiernos afines o incluso de la ayuda humanitaria cuando se desvía la finalidad de su uso ${ }^{73}$ Münkler igualmente definirá estas nuevas formas de financiación como los canales oscuros de la financiación, en los que incluye el tráfico de drogas, de armas y de todo tipo de mercancías ilegales con la finalidad de prolongar las guerras de "baja intensidad". ${ }^{74} \mathrm{En}$ esta misma línea de pensamiento, algunos autores como David Keen profundiza en los incentivos económicos de la guerra, y sostiene que podría ser más beneficioso para ciertas partes prolongar el conflicto con tal de mantener el control de los recursos económicos o posiciones de poder. Esto puede llegar a ser normal en los conflictos intraestatales o en guerras civiles dentro estados débiles donde se privatiza la violencia ${ }^{75}$.

\subsection{Emergencias complejas}

El concepto de emergencia compleja surgió como consecuencia de la irrupción de los conflictos intraestatales. Fue acuñado a finales de los años ochenta por las Naciones Unidas en un intento de diferenciar las consecuencias provocadas por este tipo de conflictos de las consecuencias de las catástrofes naturales. La emergencia compleja produce desplazamientos de personas, hambrunas, crisis sanitarias y violaciones de Derechos Humanos. Estos acontecimientos apremiaron tanto a una respuesta internacional de carácter humanitario como a una presencia militar externa.

De este modo Bellamy, aunque adhiriéndose a las tesis de las "nuevas guerras", centra sin embargo su aportación en la respuesta internacional que se proporciona por parte de la comunidad internacional a este tipo de conflicto. Coincide con Kaldor en la relación directa de estos nuevos conflictos con la globalización, en el incremento de la preeminencia de nuevos actores, el predominio de la identidad política y en las nuevas formas de violencia. ${ }^{76}$ Estas diferencias son las que desencadenan que estas guerras requieran de respuestas, al empezarse a producir una brecha creciente entre las tareas que se requería efectuar y los

\footnotetext{
${ }^{71}$ Véase Kaldor (2001), op. cit., p.119-144.

72 Duffield lo llama "asset transfer" con la acepción de asset que en inglés incluye todo tipo de recursos, entendiendo éstos como activos financieros, medios, materiales, etc. Véase Duffield, Mark (1994): "The Political Economy of Internal War: Asset Transfer, Complex Emergencies, and International Aid," en Macrae, J. \& Zwi, A. (eds.) (1994): War \& Hunger: Rethinking International Responses to Complex Emergencies, London, Zed Books.

${ }^{73}$ Véase Kaldor (2001), op. cit.

${ }^{74}$ Véase Münkler, op. cit.

${ }^{75}$ Keen, David: "Incentives and Disincentives for Violence", en Berdal, M. y Malone, D. (2000): Greed and Grievance: Economic Agendas in Civil Wars, Boulder, CO., Lynne Rienner Publishers Inc, pp. 19-43.

${ }^{76}$ Véase Bellamy, op. cit., p. 31.
} 
medios de los que se disponía. Y ello significó que los viejos principios de las Operaciones de Paz ya no eran útiles para los actuales conflictos. Destaca Bellamy que las "nuevas guerras" han producido emergencias complejas en las que participan muchos actores externos militares y civiles. Con el concepto de emergencia compleja surge el problema de las respuestas prácticas a las consecuencias humanitarias de las "nuevas guerras". Destaca que este tipo de guerras han suscitado una respuesta internacional: una respuesta por parte de la comunidad humanitaria y de la militar. Es a partir de estos conflictos cuando aparece la necesidad de interoperabilidad de los militares con las ONG y las Agencias de Naciones Unidas. Bosnia y Kosovo marcaron los puntos de inflexión. El esfuerzo de coordinación que se llevó a cabo fue ingente debido a la presencia de miles de ONG en el terreno.

En este contex to de presencia de actores transnacionacionales, Münkler nos proporciona a su vez las claves que explican el creciente interés de estos actores para intervenir militarmente en las "nuevas guerras":

a) La brutalidad de las estrategias, justifica la intervención militar con la finalidad de proteger los Derechos Humanos, inhibiendo así el estallido de "nuevas guerras" en otros lugares.

b) La importancia de los recursos naturales en las "nuevas guerras".

c) La larga duración de las "nuevas guerras" que justifica la intervención militar para poner fin a la lucha

d) El elevado coste de efectos tales como los flujos de refugiados, la propagación de la violencia, actividades ilegales y de las economías de guerra que desestabilizan los países vecinos o regiones enteras. ${ }^{77}$

Esto también afecta el grado de participación de las organizaciones humanitarias, las diásporas, los actores y las redes delictivas, las compañías militares privadas, mercenarios o las fuerzas de mantenimiento de la paz internacional. Estas repercusiones implican una presencia internacional que incluye como se ha apuntado a todo un "ejército" internacional de actores. $^{78}$

Por último Duffield en este contexto de respuestas adecuadas a emergencias complejas apunta a que este tipo de guerras tienen unas causas económicas, sociales y políticas que requieren de la respuesta más adecuada a las mismas. Según este autor, la guerra ya no es un asunto de estado a la manera clausewitziana. Es un problema de subdesarrollo y de crisis política, y como tal, necesita profesionales del desarrollo y de la seguridad para encontrar nuevas formas de trabajo en equipo. La fusión entre la seguridad y desarrollo se ha facilitado por las instituciones mediante la privatización y la subcontratación de las responsabilidades del estado. De esta forma, comenzaron a surgir redes que unen a los Gobiernos, a las ONG, al sector empresarial que han facilitado las acciones de respuesta. De hecho, se han identificado cinco comunidades cuya coordinación es necesaria para una respuesta exitosa a las emergencias complejas. Estas comunidades que se han involucrado en los nuevos modos de perseguir la paz liberal son: las ONG, los Gobiernos donantes, las agencias multilaterales, el establishment militar y el sector empresarial. En su acomodación al nuevo entorno de la seguridad en los noventa, las partes relevantes de cada una de estas comunidades, han estado sujetas a importantes procesos de cambio y adaptación y no sólo en lo que se refiere a su organización interna y política. Bajo la influencia de la fusión de desarrollo y seguridad, y la

\footnotetext{
${ }^{77}$ Véase Münkler, op. cit., pp. 125-130.

${ }^{78}$ Véase Kaldor (2001), op. cit., pp. 15-18, 145.
} 
privatización de estas responsabilidades, las conexiones y las redes transfronterizas se han consolidado, e incluso han surgido otras formas nuevas de colaboración. ${ }^{79}$

\subsection{Marco geográfico}

Desde la aparición del estado westfaliano, las guerras se han disputado fundamentalmente entre estados, esto es, se ha tratado de conflictos interestatales si bien en algunas ocasiones han acontecido en el interior de los estados en el marco de procesos de descolonización o emancipación de la metrópolis (como por ejemplo en Sudamérica). Posteriormente en el siglo $\mathrm{XX}$, el marco regional de los conflictos pasó en algunas ocasiones a guerras de orden global (I y II Guerra Mundial). Por tanto, en sentido clásico, las guerras acontecidas hasta el final de la Guerra Fría han de ser entendidas fundamentalmente bien como internas en procesos de descolonización, bien como interestatatales. Sin embargo, después de la Guerra Fría y en relación con la naturaleza geográfica en el que se desarrollan, los diversos autores que se postulan a favor de la existencia de las "nuevas guerras" como Snow, Kaldor, Duffield, Münkler y Bellamy coinciden en que estos nuevos conflictos quedan encuadrados en la categoría de los conflictos intraestatales.

Así, este tipo de conflictos ha ganado sustancial y progresivamente en número a las guerras interestatales. De los 31 conflictos que acontecieron entre 1990 y 1997, 24 de ellos encajaron en la categoría de intraestatales ${ }^{80}$, mientras que solo 3 conflictos de los que tuvieron lugar en el periodo del 2000 al 2010 entran en la tipología de interestatales. ${ }^{81}$ En el año 2012 había 23 conflictos intraestatales y un conflicto interestatal. En el año 2013, 24 conflictos eran intraestatales, uno más que en $2012^{82}$. Esta tipología está casi desapareciendo por diversos motivos. Creveld por ejemplo considera que las guerras interestatales son escasas o casi inexistentes debido a que armamento nuclear ha transformado los conflictos entre estados en algo sin sentido. A esto se le añaden por un lado que las instituciones internacionales y la normativa legal para evitar las guerras como instrumento legítimo de la política, conviertan los conflictos convencionales entre los estados en irrelevantes. ${ }^{83}$

\subsection{Marco legítimo-legal}

El marco legítimo-legal de los nuevos conflictos ha sufrido cambios sustanciales. Podemos señalar como elementos novedosos o identificativos, la erosión del monopolio de la violencia por parte del estado, lo que ha ocasionado que se extienda el barbarismo como método empleado en el ejercicio de la fuerza.

El monopolio de la fuerza o de la violencia legítima pertenece a los elementos esenciales del estado moderno. Así desde Thomas Hobbes y sobre todo Max Weber, se define el estado como una organización política cuyo cuerpo administrativo y funcionarial detenta de forma exitosa el monopolio del uso legítimo de la fuerza física en la aplicación de sus leyes. ${ }^{84}$

Autores como Cooper clasifican el mundo en tres categorías dependiendo del grado de ejercicio del monopolio de la violencia por parte del estado. Establece el mundo pre-moderno,

\footnotetext{
${ }^{79}$ Véase Duffield (1994), op. cit., pp. 76-84.

${ }^{80}$ Sarkees, Meredith; Wayman, Frank W. y Singer J. David: "Inter-State, Intra-State, and Extra-State Wars: A comprehensive Look at Their Distribution over Time, 1816-1997", International Studies Quarterly, vol. 47, nº 1 (2003), pp. 49-70.

${ }^{81}$ García, Caterina: "Las "nuevas guerras" del siglo XXI. Tendencias de la conflictividad armada contemporánea", ICPS, Working Paper, nº 323 (2013), en http://www.icps.cat/archivos/Workingpapers/wp323.pdf.

${ }_{82}$ Para una mayor información ver los datos del Uppsala Conflict Data Programme (UCDP): http://www.pcr.uu.se/research/ucdp/datasets/.

${ }^{83}$ Véase Van Creveld, op. cit.

${ }^{84}$ Weber, Max (2012): El político y el científico, Madrid, Alianza Editorial.
} 
en el que los estados no son capaces de imponer el monopolio legítimo del uso de la fuerza como Somalia, Afganistán o Liberia. La debilidad del estado permite el surgimiento de actores no estatales como terroristas, grupos criminales, carteles de drogas, etc. Una segunda tipología son los estados dentro del mundo moderno, es decir, aquellos estados que detentan ese monopolio como herramienta para equilibrar el poder y como base de su seguridad. Rusia, India y China son un ejemplo. Por último el mundo post-moderno que representa un sistema en el que los estados han implosionado a través de la creación de regímenes supranacionales. Aquí la limitación a la soberanía no genera caos, sino que estimula el orden. La Unión europea es un ejemplo. ${ }^{85}$

Sin embargo, la aparición de los llamados estados fallidos o colapsados ha erosionado este monopolio de la violencia sobre su territorio y sobre los ciudadanos administrados en su defensa, por lo que la aplicación de la ley se hace complicada o imposible ${ }^{86}$. La imposibilidad del estado en detentar este ejercicio, que diversos autores atribuyen a la globalización y la interconexión mundial ${ }^{87}$ viene acompañada de una pérdida del control sobre los instrumentos que ejercen la coerción lo que origina una privatización de la violencia. ${ }^{88}$

En otras palabras, siempre y cuando el estado era el "Maestro de la guerra", la guerra se llevaba a cabo dentro de un conjunto de reglas, el ius in bello, con el resultado de lo Münkler llama la "contención" de la violencia en las guerras. ${ }^{89} \mathrm{Si}$ el estado por el contrario, pierde su posición como director de la guerra, tenemos que esperar una incontrolada escalada de violencia con una difuminación de la distinción tradicional entre la guerra, la guerra de guerrillas, el crimen organizado, los actos terroristas, y grandes violaciones masivas de los Derechos Humanos. $^{90}$

Kaldor afirma que dicho monopolio también lo erosiona la transnacionalización de las fuerzas militares, que comenzó durante las dos guerras mundiales y quedó institucionalizada por la política de bloques de la Guerra Fría y las relaciones transnacionales que debilitan la capacidad de los estados para usar la fuerza de modo unilateral. También a ello coadyuvan razones prácticas, como son el creciente poder destructivo de la tecnología militar y la mayor interconexión entre los estados, sobre todo en el ámbito militar. Además, las alianzas militares, la producción y el comercio internacional de armas, diversas formas de cooperación e intercambio militar, los acuerdos de control de armamento, han creado una forma de integración militar mundial ${ }^{91}$

Esta erosión del exclusivo uso de la violencia por parte del estado, ha resultado en la existencia de una mayor privatización de la violencia. Es esta la que podemos considerar como otra de las señas de identidad de las "nuevas guerras". Esta creciente dependencia de los actores no estatales para realizar tareas militares y de seguridad en el contexto de los

\footnotetext{
${ }^{85}$ Cooper Robert (2003): The Breaking of Nations: Order and Chaos in the Twenty-first Century, London, New York: Atlantic Books.

${ }^{86}$ Los estados fallidos pueden estudiarse de forma empírica gracias al Failed State Index elaborado anualmente por el Fund for Peace (FFP). Según este índice, para el año 2014 habría 5 países en muy alta alerta; 11 países en alta alerta; 18 en alerta. Luego habría una serie de países sobre los que se pone en aviso para finalmente completar el ranking con 40 países que estarían entre los estables o muy estables. Ver http://ffp.statesindex.org/.

${ }^{87}$ Véase Kaldor (2001), op. cit.

${ }^{88}$ Ibid.

${ }^{89}$ Véase Münkler, op. cit.

${ }^{90}$ Véase Kaldor (2001), op. cit. y Münkler, op. cit.

${ }^{91}$ Véase Kaldor (2001), op. cit.
} 
conflictos armados se encuentra en la literatura académica con las denominaciones de privatización de la guerra o simplemente como privatización de la violencia ${ }^{92}$.

La violencia se privatiza cada vez más, tanto como resultado de la creciente delincuencia organizada y la aparición de los grupos paramilitares, como de la desaparición de legitimidad política. El resultado es una profunda confusión entre los papeles del soldado y el de los criminales ${ }^{93}$. En sentido parecido se manifiesta Mueller cuando afirma que la guerra se está convirtiendo en algo obsoleto y lo que queda son matones como "combatientes residuales" 94 . Según él, lo que ocurre en las guerras de hoy es que se caracterizan por el oportunismo y el choque entre matones, más que por el choque de civilizaciones o de programas políticos, si bien los implicados proclaman astutamente que las causas son de otro orden para con ello justificar sus actividades, puesto que poner el acento sobre la depredación y el saqueo no sería políticamente correcto ${ }^{95}$. Todo ello origina que se haga difícil distinguir entre criminalidad y conflicto armado, es decir entre crimen organizado y guerra.

Así, la privatización de la violencia es vista como un indicador de la evolución del papel del estado en la guerra, y una ruptura de las fronteras entre lo público y lo privado (y entre militares y civiles) y por tanto, como una clara desviación de la función central del estado en el marco clausewitziano. La privatización es un signo de la incapacidad del estado para asegurar o mantener un "monopolio de la fuerza militar" y contribuye a la "desestatalización " de la guerra ${ }^{96}$. La privatización de la guerra, pone de relieve el hecho de que los ejércitos regulares operarán junto a grupos militares privados que pueden ser utilizados por cualquier persona que está dispuesto a pagar sus servicios. ${ }^{97}$

Como consecuencia de la pérdida del monopolio de la violencia por parte del estado y la privatización de la guerra, se ha socavado la eficacia del Derecho Internacional, esto es, El ius ad bellum y ius in bello destinados a evitar la guerra o las consecuencias de la misma a través de normas y prácticas humanitarias comunes ${ }^{98}$. En consecuencia, las "nuevas guerras" eluden en gran medida estos marcos legales, toda vez que ya no se trata de guerra entre los estados en la que los adversarios se reconocen unos a otros como enemigos "legítimos". En estos nuevos conflictos los nuevos actores no estatales se caracterizan por las nociones de reciprocidad de ilegitimidad. ${ }^{99}$ Es lo que podemos denominar como barbarismo.

En consecuencia, el colapso estatal que se produce en muchos estados con la consiguiente incapacidad para ejercer el monopolio de la fuerza conduce, como se ha apuntado, a la falta de respeto durante los conflictos por el derecho y usos de la guerra. Es decir el llamado ius in bello no es respetado como consecuencia de la usurpación de ese monopolio por grupos privados que no respetan unas normas y una legalidad que conforma un

\footnotetext{
${ }^{92}$ Véase por ejemplo, Kaldor (2001), op. cit.; Cilliers, Jakkie y Mason, Peggy (1999): Peace, profit or plunder? : the privatisation of security in war-torn African societies, Halfway House: Institute for Security Studies; Mueller, John: "Simplicity and Spook: Terrorism and the Dynamics of Threat Exaggeration", International Studies Perspectives, vol. 6, n 2 (2005), pp. 208-234; Mandel, Michael: " Politics and Human Rights in the International Criminal Tribunal for the Former Yugoslavia : our Case Against NATO and the Lessons To Be Learned from it" (2002) ; Münkler, op. cit.; Avant, Deborah (2005): The market for force : the consequences of privatizing security, Cambridge, Cambridge University Press.

${ }^{93}$ Véase Kaldor (2001), op. cit.

${ }^{94}$ Véase Mueller, op. cit.

${ }^{95}$ Ibid.

${ }^{96}$ Una de las características principales de los llamados estados fallidos (término acuñado por Madeleine Albright ex-Secretaria de Estado de los EEUU), como Somalia o actualmente República Centroafricana, es la incapacidad de detentar y ejercer el monopolio de la violencia.

${ }^{97}$ Véase Münkler, op . cit.

${ }^{98}$ Convenios de Ginebra y protocolos adicionales y Convenio de la Haya.

${ }^{99}$ Véase Münkler, op. cit. .
} 
sistema internacional hecho por los estados y por tanto acomodado al modelo westfaliano de "viejas guerras". Así se manifiestan múltiples autores quienes señalan que en las "nuevas guerras" se ignora todo el cuerpo legislativo que regulariza la violencia. ${ }^{100}$ Bellamy señala que este nuevo barbarismo también es un instrumento usado por los nuevos actores para asegurar la lealtad al grupo. ${ }^{101}$

Así, Kaldor explica que la extrema violencia de las "nuevas guerras" en las que se incluyen las violaciones sistemáticas, los asesinatos masivos, la limpieza étnica o las hambrunas forzadas mediante los sitios a las poblaciones, cae dentro de la definición de genocidio con arreglo a la Convención de Ginebra de 1948. Por tanto las "nuevas guerras" se asocian a la violación sistemática del Derecho Internacional, en la que la línea entre la guerra, el crimen organizado y las violaciones de los derechos humanos a gran escala queda muy desdibujada. ${ }^{102}$ Es lo que Shaw define como "guerras degeneradas"103, en las que la descomposición de las estructuras estatales corre en paralelo con el uso de las técnicas de guerrillas y actos terroristas de forma indiscriminada.

\section{Operacionalización de las variables identificativas de las "nuevas guerras"}

Las variables independientes estudiadas en el apartado anterior, son susceptibles de ser medidas, analizadas o evaluadas, tanto cualitativa como cuantitativamente. La estrategia que se adopta es una estrategia bipolar: (i) "nueva guerra" u (ii) otro tipo de guerras. Por ello, la respuesta es dicotómica (SI/NO). Esto significa que si los resultados que se obtienen son mayoritariamente positivos (más respuestas síes que noes) estaremos ante un estudio de caso de "nueva guerra".

Como se puede apreciar y para una mayor claridad, organización y comprensión, se ofrecen dos tablas. En la primera se elabora una síntesis de la categoría, la variable y el autor que estudia dicha categoría. En la segunda tabla, que es propiamente la herramienta teóricometodológica construida, se proporcionan las categorías, las variables, los indicadores, la respuesta y la fuente posible donde encontrar la información.

En la tabla 1 podemos ver un resumen de las categorías, las variables y los autores que más se han centrado en esas características.

1. Actores

2. Causas

a. Multiplicidad y fraccionamiento de los actores internos

a. Identidades religiosas, étnicas, tribales.

b. Contienda por los recursos. Codicia

3. Métodos y estrategias

a. Violencia contra los civiles

b. Asimetría

c. Hibridismo

d. Diferente tolerancia a las bajas

e. Terrorismo

4. Formas de financiación

a. Nueva economía de guerra

\footnotetext{
${ }^{100}$ Véase por ejemplo, Holsti, op. cit.; Kaldor (2001), op. cit.; Münkler, op. cit.

${ }^{101}$ Véase Bellamy, op. cit.

102 Véase Kaldor (2001), op. cit.

${ }^{103}$ Véase Shaw, "War and globality...", op. cit.
} 
5. Emergencias complejas (internacionalización del conflicto)

a. Respuesta humanitaria

b. Respuesta militar

6. Marco geográfico

a. Intraestatal

7. Marco legítimo legal

a. Erosión monopolio de la violencia del estado. Privatización de la violencia

b. Barbarismo

Tabla 1: Características/Variables de las "nuevas guerras"

\begin{tabular}{|c|c|c|}
\hline CATEGORIA & VARIABLE & AUTORES \\
\hline ACTORES & $\begin{array}{l}\text { Multiplicidad y fraccionamiento } \\
\text { de los actores internos }\end{array}$ & $\begin{array}{l}\text { Kaldor, Snow, Eppler, Münkler, } \\
\text { Holsti, Duffield, Heupel, Zangl }\end{array}$ \\
\hline \multirow{2}{*}{ CAUSAS } & $\begin{array}{l}\text { Identidades religiosas, étnicas, } \\
\text { tribales. }\end{array}$ & $\begin{array}{l}\text { Kaldor, Van Creveld, Münkler, } \\
\text { Collier, Hoeffler, Bellamy, } \\
\text { Enzensberger }\end{array}$ \\
\hline & $\begin{array}{l}\text { Contienda por los recursos } \\
\text { Codicia }\end{array}$ & Keen, Münkler Collier, Hoeffler \\
\hline \multirow{5}{*}{$\begin{array}{l}\text { METODOS } \\
\text { Y } \\
\text { ESTRATEGIAS }\end{array}$} & Violencia contra los civiles & Kaldor, Smith \\
\hline & Asimetría, & $\begin{array}{l}\text { Holsti, Rice, Bacevich, Münkler, } \\
\text { Yost, Duffield }\end{array}$ \\
\hline & Hibridismo & Hoffman \\
\hline & Terrorismo & Kaldor, Münkler \\
\hline & Diferente tolerancia a las bajas & Münkler, Luttwak \\
\hline FORMAS DE FINANCIACION & Nueva economía de guerra & Kaldor, Münkler. \\
\hline \multirow[t]{2}{*}{ EMERGENCIAS COMPLEJAS } & Respuesta humanitaria & \multirow{2}{*}{$\begin{array}{l}\text { Kaldor, Münkler, Bellamy, } \\
\text { Duffield }\end{array}$} \\
\hline & Respuesta militar internacional & \\
\hline MARCO GEOGRAFICO & $\begin{array}{l}\text { Global } \\
\text { Regional } \\
\text { Interestatal }^{104} \\
\text { Intraestatales. }\end{array}$ & $\begin{array}{l}\text { Snow, Kaldor, } \quad \text { Duffield, } \\
\text { Münkler, Van Creveld, Uppsala }\end{array}$ \\
\hline \multirow[t]{2}{*}{ MARCO LEGITIMO-LEGAL } & $\begin{array}{l}\text { Erosión monopolio de la } \\
\text { violencia del estado. }\end{array}$ & $\begin{array}{lrr}\text { Kaldor, } & \text { Duffield, } & \text { Münkler, } \\
\text { Cooper. } & \text { Cilliers, } & \text { Mason, } \\
\text { Mueller, } & \text { Mandel, } & \text { Münkler, } \\
\text { Avant, Eppler, Singer } & \end{array}$ \\
\hline & Barbarismo & $\begin{array}{l}\text { Kaldor, Holsti, } \quad \text { Münkler, } \\
\text { Bellamy, Shaw }\end{array}$ \\
\hline
\end{tabular}

Fuente: Elaboración propia

En la tabla 2 se ha procedido a la operacionalización de las variables identificativas de las "nuevas guerras" en indicadores medibles y analizados en el apartado anterior que nos puedan

${ }^{104}$ Las nuevas guerras no abarcan conflictos interestatales, sin embargo, incluimos esta categoría porque con nuestra herramienta tratamos de determinar si es o no nueva guerra. 
aportar un resultado positivo o negativo en el grado de cumplimiento de cada una de las mismas. En la columna de la derecha se proporcionan ejemplos de fuentes que pueden consultarse para la investigación. Dependiendo de la calidad y cantidad de fuentes consultadas la opción por un resultado u otro disfrutará de una mayor argumentación para evaluar si se trata de un caso o no de "nueva guerra".

Las fuentes que se pueden utilizar para conducir los estudios de caso en las investigaciones pertinentes recurren a fuentes abiertas en internet de medios de comunicación, think tank ${ }^{105} \mathrm{e}$ índices especializados, fundamentalmente. Los índices cuantitativos elaborados por diferentes centros de investigación con los que se puede trabajar y que ponemos como ejemplo, son: Failed State Index (Fund For Peace); Freedom House, Transparencia Internacional, Berterlsmann Transformation Index (Beterlsmann Stiftung), Rule of Law Index (World Justice Project). También se puede trabajar con fuentes de centros de investigación de universidades especializadas en estas materias como el Uppsala Data Programme de la Universidad de Uppsala (Suecia), así como datos proporcionados por Human Rights Watch u ONG como Amnistia Internacional. También son fuentes interesantes la documentación e informes producidos por OOII como Naciones Unidas y sus agencias, la UE, el Banco Mundial o la OTAN.

Tabla 2: Operacionalización de las características de las "nuevas guerras"

\begin{tabular}{|c|c|c|c|c|}
\hline Categoría & Variable & Indicador & Resultado & Fuente $^{106}$ \\
\hline ACTORES & $\begin{array}{l}\text { Multiplicidad y } \\
\text { fraccionamiento } \\
\text { de actores } \\
\text { internos }\end{array}$ & $\begin{array}{l}\begin{array}{l}\text { Fuerzas armadas } \\
\text { regulares }\end{array} \\
\begin{array}{l}\text { Grupos } \\
\text { paramilitares }\end{array} \\
\begin{array}{l}\text { Unidades de auto- } \\
\text { defensa. }\end{array} \\
\begin{array}{l}\text { Rebeldes, grupos } \\
\text { insurgentes }\end{array} \\
\text { Grupos terroristas } \\
\text { Señores de la guerra }\end{array}$ & $\begin{array}{l}\text { SI/NO } \\
\text { SI/NO } \\
\text { SI/NO } \\
\text { SI/NO } \\
\text { SI/NO } \\
\text { SI/NO }\end{array}$ & $\begin{array}{l}\text { Medios comunicación, } \\
\text { Think, tanks, UN, } \\
\text { fuentes } \\
\text { gubernamentales }\end{array}$ \\
\hline
\end{tabular}

105 Para conocer los principales think tank del mundo se puede consultar la siguiente página web: http://www.ecologic.eu/sites/files/news/2014/gotoreport2013_final.pdf.

${ }^{106}$ Para la fuente de medios de comunicación ver los diferentes pies de página. 


\begin{tabular}{|c|c|c|c|c|}
\hline \multirow[t]{2}{*}{ CAUSAS } & $\begin{array}{l}\text { Identidades } \\
\text { religiosas, de } \\
\text { clan o } \\
\text { lingüísticas. }\end{array}$ & $\begin{array}{l}\text { Clan } \\
\text { Lengua } \\
\text { Religión } \\
\text { Otredad }\end{array}$ & $\begin{array}{l}\text { SI/NO } \\
\text { SI/NO } \\
\text { SI/NO } \\
\text { SI/NO }\end{array}$ & $\begin{array}{lr}\text { Middle East } & \text { Policy } \\
\text { Council. } & \\
\text { Medios } & \\
\text { comunicación } & \\
\text { Think tanks } & \\
\end{array}$ \\
\hline & $\begin{array}{l}\text { Contienda por } \\
\text { los recursos. } \\
\text { Codicia. }\end{array}$ & $\begin{array}{l}\text { Posibilidad de } \\
\text { beneficios } \\
\text { económicos }\end{array}$ & SI/NO & $\begin{array}{l}\text { Think tanks } \\
\text { Medios comunicación } \\
\text { ONG }\end{array}$ \\
\hline \multirow[t]{3}{*}{$\begin{array}{l}\text { METODOS Y } \\
\text { ESTRATEGIAS }\end{array}$} & $\begin{array}{l}\text { Violencia contra } \\
\text { los civiles }\end{array}$ & $\begin{array}{l}\text { Bajas civiles } \\
\text { Desplazamientos } \\
\text { forzados de la } \\
\text { población }\end{array}$ & $\begin{array}{l}0-20 \% \\
20-40 \% \\
40-60 \% \\
60-80 \% \\
80-100 \% \\
\text { SI/NO } \\
\text { Ver cifras } \\
\text { Ver cifras }\end{array}$ & $\begin{array}{l}\text { Medios de } \\
\text { comunicación } \\
\text { Human Rights Watch } \\
\text { Refugees International } \\
\text { ACNUR } \\
\text { Amnistía Internacional }\end{array}$ \\
\hline & Asimetría & $\begin{array}{l}\text { Disimilitud en: } \\
\text { Armamento y } \\
\text { medios. } \\
\text { Grado de } \\
\text { instrucción y } \\
\text { adiestramiento de } \\
\text { las unidades } \\
\text { Recursos } \\
\text { económicos } \\
\text { disponibles y } \\
\text { Logística } \\
\\
\text { Capacidad de } \\
\text { reclutamiento }\end{array}$ & $\begin{array}{l}\text { SI/NO } \\
\text { SI/NO } \\
\text { SI/NO } \\
\text { SI/NO } \\
\text { SI/NO }\end{array}$ & $\begin{array}{l}\text { SIPRI } \\
\text { Medios comunicación } \\
\text { World Peace } \\
\text { Foundation }\end{array}$ \\
\hline & Hibridismo & $\begin{array}{l}\text { Coexistencia armas } \\
\text { de alta precisión } \\
\text { con armamento } \\
\text { convencional } \\
\text { obsoleto. } \\
\text { Concurrencia } \\
\text { combates } \\
\text { convencionales con }\end{array}$ & $\mathrm{SI} / \mathrm{NO}$ & $\begin{array}{l}\text { SIPRI } \\
\text { Institute for the Study } \\
\text { of War. }\end{array}$ \\
\hline
\end{tabular}




\begin{tabular}{|c|c|c|c|c|}
\hline & & $\begin{array}{l}\text { tácticas insurgentes } \\
\text { Dificultad de } \\
\text { distinguir entre } \\
\text { combatientes no } \\
\text { combatientes }\end{array}$ & & Medios comunicación \\
\hline & $\begin{array}{l}\text { Diferente } \\
\text { tolerancia a las } \\
\text { bajas }\end{array}$ & & $\begin{array}{l}\text { ALTA } \\
\text { MEDIA } \\
\text { BAJA }\end{array}$ & $\begin{array}{l}\text { Medios comunicación } \\
\text { OTAN } \\
\text { Encuestas de opinión } \\
\text { pública }\end{array}$ \\
\hline & Terrorismo & & SI/NO & $\begin{array}{ll}\text { Medios } & \mathrm{de} \\
\text { comunicación } & \\
\text { Failed State Index }\end{array}$ \\
\hline $\begin{array}{l}\text { FORMAS DE } \\
\text { FINANCIACION }\end{array}$ & $\begin{array}{l}\text { Nueva economía } \\
\text { de guerra }\end{array}$ & $\begin{array}{l}\text { Saqueo, Robo y } \\
\text { Pillaje } \\
\text { Extorsión, } \\
\text { Corrupción } \\
\text { Ayuda exterior } \\
\text { Mercado negro }\end{array}$ & $\begin{array}{l}\mathrm{SI} / \mathrm{NO} \\
\mathrm{SI} / \mathrm{NO} \\
\mathrm{SI} / \mathrm{NO} \\
\mathrm{SI} / \mathrm{NO} \\
\mathrm{SI} / \mathrm{NO}\end{array}$ & $\begin{array}{l}\text { Medios } \\
\text { Comunicación. } \\
\text { Transparency } \\
\text { International Index } \\
\text { Berterlsmann } \\
\text { Transformation Index } \\
\text { (BTI) } \\
\text { Carnegie Endowment } \\
\text { International for Peace. } \\
\text { Human Rights Watch } \\
\text { Amnistía Internacional }\end{array}$ \\
\hline \multirow{2}{*}{$\begin{array}{l}\text { EMERGENCIA } \\
\text { COMPLEJA }\end{array}$} & $\begin{array}{l}\text { Respuesta } \\
\text { humanitaria }\end{array}$ & $\begin{array}{l}\text { ONG, expatriados } \\
\text { voluntarios, } \\
\text { Agencias de } \\
\text { Naciones Unidas }\end{array}$ & $\begin{array}{l}\text { SI/NO } \\
\text { SI/NO }\end{array}$ & \multirow{2}{*}{$\begin{array}{l}\text { ACNUR } \\
\text { Amnistía Internacional } \\
\text { Human Right Watch } \\
\text { Cruz Roja } \\
\text { ONG } \\
\text { SIPRI, PRIO, NUPI... }\end{array}$} \\
\hline & $\begin{array}{l}\text { Respuesta } \\
\text { militar } \\
\text { internacional }\end{array}$ & $\begin{array}{l}\text { Fuerzas regulares } \\
\text { extranjeras bajo } \\
\text { auspicios } \\
\text { internacionales } \\
\text { Mercenarios } \\
\text { extranjeros } \\
\text { Intervención de } \\
\text { fuerzas regulares } \\
\text { extranjeras al } \\
\text { margen del derecho } \\
\text { internacional }\end{array}$ & $\begin{array}{l}\text { SI/NO } \\
\text { SI/NO } \\
\text { SI/NO }\end{array}$ & \\
\hline $\begin{array}{l}\text { MARCO } \\
\text { GEOGRAFICO }\end{array}$ & Intraestatal & $\begin{array}{l}\text { Muchos estados en } \\
\text { distintos puntos del } \\
\text { planeta }\end{array}$ & $\begin{array}{l}\text { SI/NO } \\
\text { SI/NO }\end{array}$ & $\begin{array}{l}\text { Uppsala Conflict Data } \\
\text { Programme }\end{array}$ \\
\hline
\end{tabular}




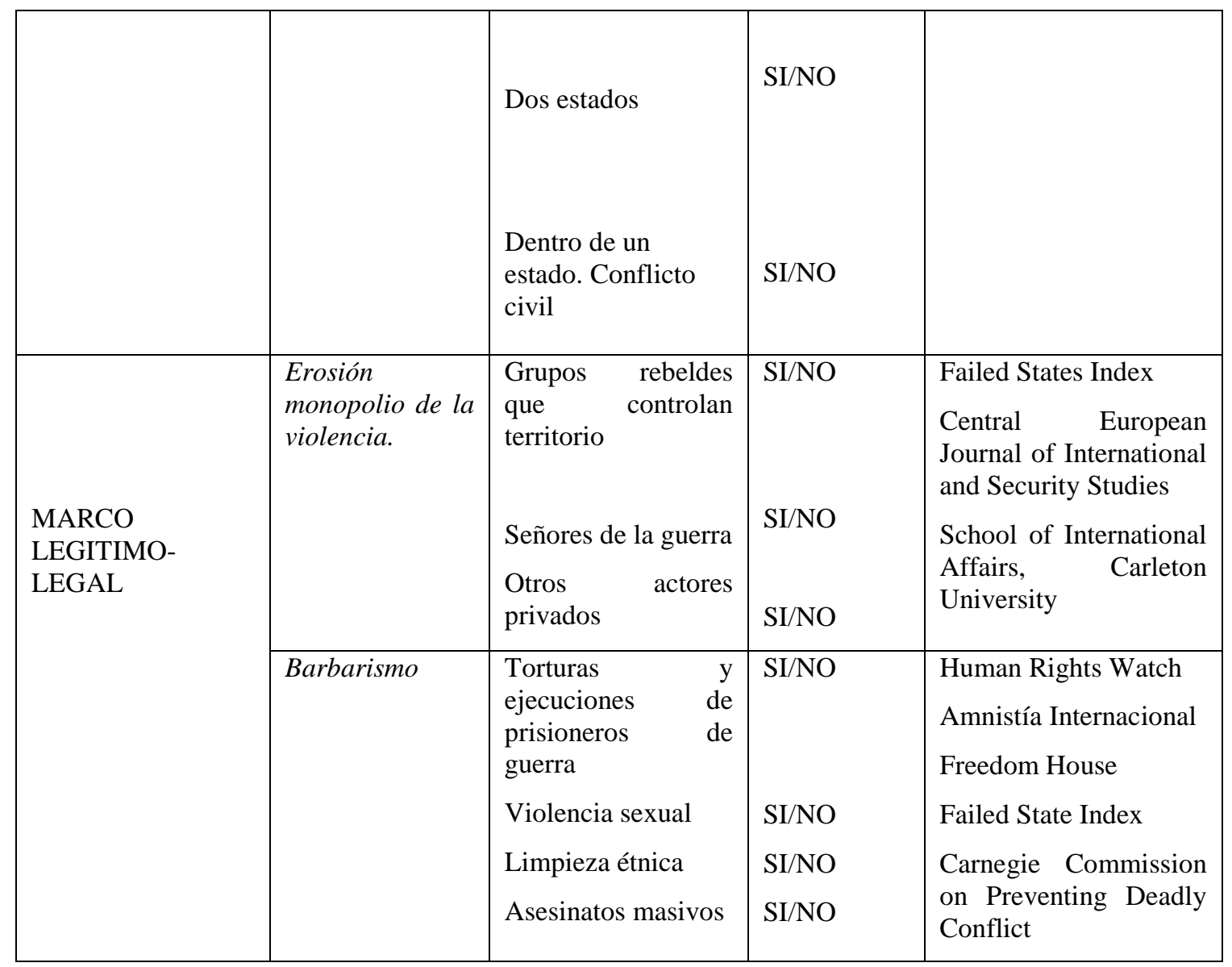

Fuente: Elaboración propia

\section{Conclusiones}

Este trabajo ha tenido como objetivo central poner de relieve el concepto de "nuevas guerras" utilizando una estrategia metodológica que tenga la facultad de llevar a cabo estudios de caso y estudios comparados de distintos conflictos. Con dicha finalidad se ha conducido una revisión de la literatura sobre las "nuevas guerras" y su aportación al campo del estudio de los conflictos contemporáneos para poder ofrecer una serie de variables explicativas que mediante su operacionalización puedan constituir una herramienta útil de trabajo que estudie pormenorizadamente los diferentes casos de conflictos. Para cada una de estas variables hemos procedido a su operacionalización en una serie de indicadores, proponiendo y haciendo acopio también de unas fuentes de consulta lo más variadas posible, donde se pueden encontrar los datos más significativos que permiten poder disponer de la información más imprescindible para estudiar las los conflictos contemporáneos.

Las principales variables identificadas sobre estas materias son las siguientes: (i) son conflictos intraestatales; (ii) las causas residen en cuestiones identitarias y la lucha por la apropiación de los recursos; (iii) presencia de multitud de actores; (iv) producen emergencias complejas (presencia de actores externos); (v) sus formas de financiación son amplias y variadas; (vi) aparecen nuevos métodos y estrategias de lucha y (vii) han tenido lugar transformaciones en el marco de referencia legítimo-legal.

De esta forma y gracias a esta síntesis aportada de variables explicativas, podemos definir las "nuevas guerras" de la siguiente forma: son aquellos conflictos que tienen lugar en 
un marco geográfico que se restringe al conflicto dentro del estado, pero con repercusiones transnacionales; las causas de estos conflictos suelen ser identitarias (religiosas, étnicas) y de contienda por los recursos. Los objetivos son coincidentes con las causas porque están relacionados con una política identitaria particularista. Hay una multiplicidad y fraccionamiento de los actores internos implicados en el conflicto (fuerzas armadas regulares, grupos criminales, unidades de autodefensa...); provocan emergencias complejas que requieren de respuesta humanitaria y de respuesta militar, conduciendo a una internacionalización del conflicto como consecuencia de la globalización y también generando como consecuencia de esta unas formas de financiación que obedecen a lo que se conoce como nueva economía de guerra (saqueo, extorsión, ayuda exterior, mercado negro). Los métodos y estrategias de lucha empleados se caracterizan por una violencia contra los civiles, asimetría en el conflicto, hibridismo y diferente tolerancia a las bajas y por último, lo que denominamos el marco legitimo-legal de estos conflictos, que se caracteriza por la erosión del monopolio de la violencia con el desentendimiento del Derecho Internacional que conduce a prácticas y métodos de violencia gratuita en el ámbito del barbarismo. 